\title{
The cost-effectiveness of therapeutic drug monitoring for the prescription drug-based treatment of chronic myeloid leukemia
}

Rena M Conti, PhD; William V Padula, PhD; Russell V Becker, MA; and Salvatore Salamone, PhD

\section{What is already known about this subject}

- Tyrosine kinase inhibitors are the standard of care for first line and subsequent lines of therapy in patients with chronic myeloid leukemia (CML).

- Second-generation tyrosine kinase inhibitors have shown to exhibit greater effectiveness with more rapid molecular responses at standard doses than the first-generation tyrosine kinase inhibitor imatinib (IM).

- Therapeutic drug monitoring (TDM) is being increasingly used in cancer treatment. TDM recommendations have been made for various drugs including kinase inhibitors. A recent study demonstrated the use of TDM in patients with CML resulted in higher response rate with IM than in the second-generation tyrosine kinase inhibitors studies.

\section{What this study adds}

- Using a modified previously published cost-effectiveness model of first-line CML treatment, our results demonstrate TDM combined with imatinib TDM (IM TDM) is dominant over IM alone and second-generation nilotinib/dasatinib (NIL/DAS) in terms of the outcomes costs, quality-adjusted life-years (QALYs), and cost-effectiveness.

- Over 5 years versus NIL/DAS, IM TDM was $\$ 224,169$ lower per first-line responder for wholesale acquisition cost (WAC) low pricing and \$330,307 lower for Federal Supply Schedule (FSS) average pricing.

\section{ABSTRACT}

BACKGROUND: A recent study demonstrating the use of Therapeutic Drug Monitoring (TDM) in patients with chronic myeloid leukemia $(\mathrm{CML})$ resulted in a higher response rate with imatinib (IM) than demonstrated in second-generation tyrosine kinase inhibitor studies. The cost-effectiveness of TDM combined with IM (IM TDM) in first-line CML treatment has not yet been studied.

OBJECTIVES: To determine the cost-effectiveness of IM TDM for the first-line treatment of CML compared to tyrosine kinase inhibitor only treatment.
METHODS: A recently published costeffectiveness model of tyrosine kinase inhibitor-treatment in CML was modified to include IM TDM as a first-line tyrosine kinase inhibitor-based CML treatment option. Efficacy inputs for major molecular response (MMR) rates were taken from previously published studies: IM TDM 65\%, dasatinib 52\%, nilotinib 53\%. Annual tyrosine kinase inhibitor drug prices were derived from the Federal Supply Schedule (FSS) and the average and lowest wholesale acquisition costs (WAC) reported in the Red Book; the annual cost of TDM was $\$ 228$. Other input costs modeled in the original CML CEA model were updated

\author{
Author affiliations \\ Rena M Conti, PhD, Boston University, \\ Questrom School of Business, Department of \\ Markets, Public Policy, and Law, Boston, MA. \\ William V Padula, PhD, University of South \\ Carolina, School of Pharmacy, Department \\ of Pharmaceutical \& Health Economics, Los \\ Angeles, CA. Russell V Becker, MA, Russell \\ Becker Consulting, Chicago, IL. Salvatore \\ Salamone, PhD, Saladax Biomedical, \\ Bethlehem, PA.
}

AUTHOR CORRESPONDENCE: Salvatore Salamone, 484.547.0503; ssalamone@saladax.com

J Manag Care Spec Pharm 2021;27(8):1077-85

Copyright $(2021$, Academy of Managed Care Pharmacy. All rights reserved.

to 2016 US dollars using the medical service component of the Consumer Price Index. A US payer perspective was used with a 5 -year time horizon and a 3.0\% discount rate. The model compared first-line IM TDM versus IM alone, nilotinib (NIL) or dasatinib (DAS) in terms of the following outcomes: costs, quality-adjusted life-years (QALYs), and costeffectiveness (total cost/QALY). Deterministic and probabilistic sensitivity analyses were performed using all key clinical and economic parameters.

RESULTS: This study found that IM TDM dominates IM alone with $\$ 15,452$ to $\$ 36,940$ 
in savings and 0.25 higher QALYs. Using FSS, per patient total costs for IM and IM TDM were $\$ 270,905$ and $\$ 233,965$, respectively.; Using average WAC, these costs were $\$ 461,657$ and $\$ 446,205$, and using lowest WAC, these costs were $\$ 366,966$ and $\$ 350,090$. The results comparing first line using of IM TDM to NIL/DAS found that TDM IM had higher QALYs and lower costs (0.08 QALYs lower, and \$117,006 to \$172,420 savings per patient [varying by price basis]). Thus, in terms of costeffectiveness, IM TDM dominates NIL/DAS with both lower costs and higher QALYS.

CONCLUSIONS: IM TDM is a clinically and economically viable firstline treatment option for CML.

Tyrosine kinase inhibitors are the standard of care for firstline and subsequent lines of therapy in patients with chronic myeloid leukemia (CML). ${ }^{1}$ Second-generation tyrosine kinase inhibitors (DAS; Sprycel, Bristol-Myers Squibb) and nilotinib (NIL; Tasigna, Novartis Oncology) have been approved for the first-line treatment of CML in chronic phase by the US Food and Drug Administration. Second generation tyrosine kinase inhibitorts have shown greater effectiveness with more rapid molecular responses at standard doses than the first-generation tyrosine kinase inhibitor imatinib (IM, Gleevec, Glivec, Novartis International AG). ${ }^{2}$

\section{THERAPEUTIC DRUG MONITORING INTRODUCTION}

By measuring specific drugs at designated intervals in order to maintain a constant concentration in a patient's bloodstream, therapeutic drug monitoring (TDM) is being increasingly used in cancer treatment to gain optimal drug exposure. TDM recommendations have been made for various drugs including kinase inhibitors. ${ }^{3,4}$ A recent study of TDM with imatinib mesylate (IM TDM) for the treatment of CML found that cytogenetic response at 12 months was significantly improved compared to imatinib alone (IM alone) ${ }^{5}$ and a greater response rate compared to the efficacy of both imatinib and second generation tyrosine kinase inhibitors in other CML studies. ${ }^{4-6}$ Given that these second-generation tyrosine kinase inhibitors had previously shown more rapid molecular responses at standard doses compared to IM alone, the improved efficacy using IM TDM provides new clinical information when selecting a CML treatment. Thus, imatinib in combination with TDM may be a better choice for physicians treating CML patients.
After loss of patent exclusivity and generic entry, the prices charged for commonly used, orally formulated prescription drugs typically drops $60 \%$ to $90 \%$ off the brand name price. ${ }^{6}$ In 2015, brand imatinib lost patent exclusivity in the US, and in anticipation of generic imatinib, a study by Padula et al found that the use of generic imatinib would be the most cost-effective first line treatment option for CML over a range of possible prices compared to first-line NIL and DAS. ${ }^{7}$ At the time of the Padula publication, the market impact of the loss of patent exclusivity had not yet taken effect, and, thus, that model could only estimate the anticipated drug price impact for generic imatinib with modeled price drops of 0 to $40 \%$ in the first year and $70-90 \%$ decreases thereafter. A more recent real-world study of generic imatinib pricing found "smaller-than-expected priced reductions" following branded loss of exclusivity. The generic pricing was reduced $8 \%$ compared to the brand in the first year following loss of exclusivity and reduced by another $10 \%$ in the second year. ${ }^{8}$

Another US-based cost-effectiveness model of tyrosine kinase inhibitor treatments in CML was published in 2015 (Rochau). ${ }^{9}$ It considered 16 different treatment combinations using imatinib and second-generation tyrosine kinase inhibitor, and found that 2 of the combinations were costeffective. However, this study predated the introduction of generic imatinib onto the market.

With the high variability in potential pricing changes, the impact of actual, post-loss of exclusivity imatinib pricing on the cost-effectiveness of the generic option is unknown, and the results from the Padula study have not been validated with real world price changes. Nor has the impact of the increased efficacy of TDM usage with IM in the treatment of CML and potential cost-saving and costeffectiveness of TDM usage with IM in the treatment of CML been studied either.

\section{Methods}

A published cost-effectiveness model of tyrosine kinase inhibitor-based treatment for CML by Padula et al was used as the starting basis for our model. ${ }^{7}$ We retained most of the main structure, inputs, US payer perspective, and key assumptions of the original Padula model, such as the constant use and cost of non-drug medical care during the study period and that each tyrosine kinase inhibitor would command the same usage (based on MarketScan data), but made the following changes: First, the Padula model had three treatment arms: IM, NIL, and DAS, and patients entered the model in each of the arms in equal proportion. 


\section{FIGURE 1 Markov State Transition Diagram}

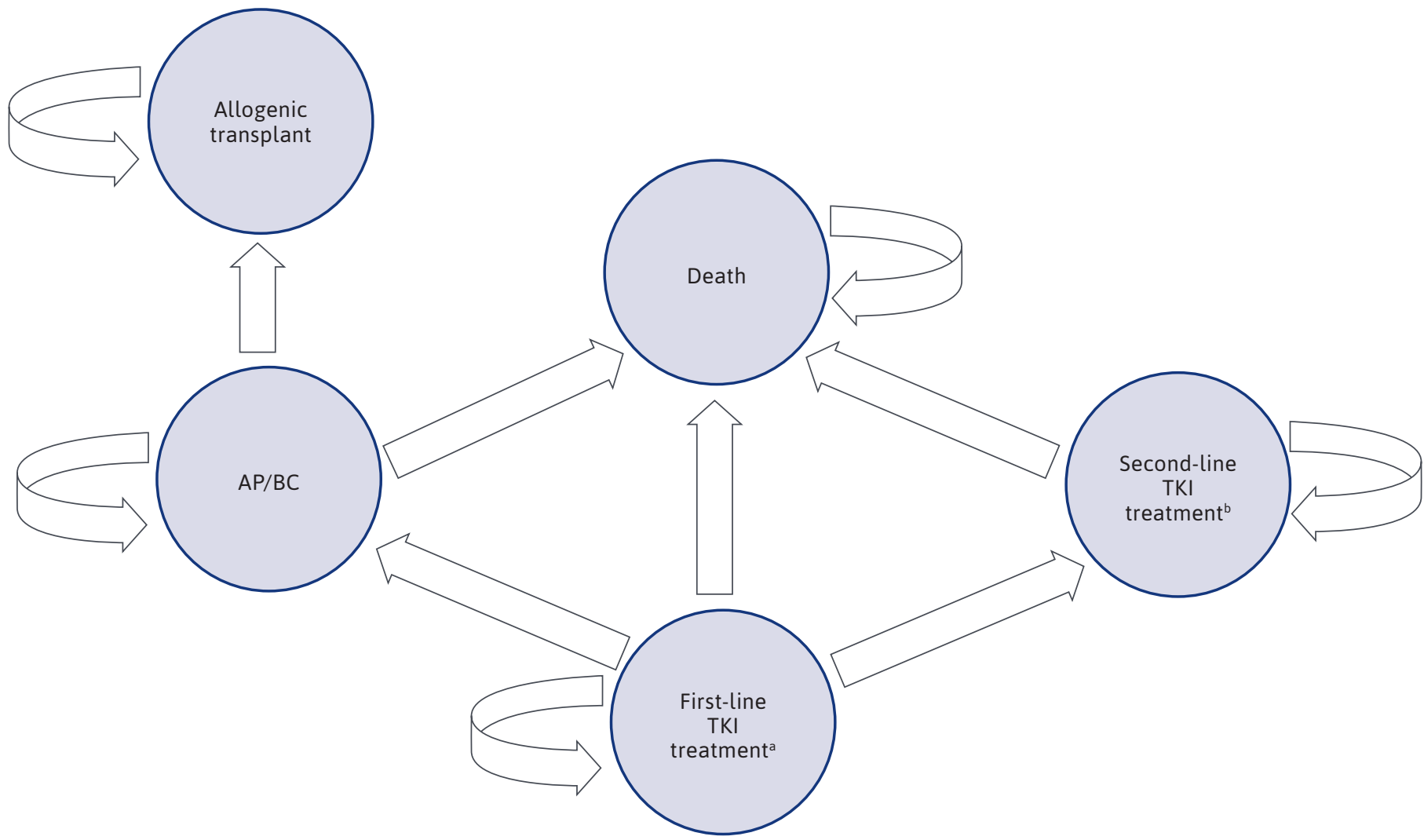

apatients enter model with a first-line TKI treatment (IM, IM TDM, DAS, NIL)

${ }^{b}$ Second-line TKI treatments vary depending on first-line treatment. Second-line NIL and DAS follow first-line IM or IM TDM. Second-line IM and DAS follow first-line NAS, and second-line IM and NIL follow first-line DAS.

$A P / B C=$ accelerated phase or blast crisis; $D A S=$ dasatinib; $I M T D M=$ imatinib with therapeutic drug monitoring; $N I L=$ nilotinib; $I M=i m a t i n i b ; T K I=T y r o s i n e ~ K i n a s e$ Inhibitor.

However, in our model, IM TDM was added as comparator arm. A half of the patients in the second-generation tyrosine kinase inhibitor comparator arm were given DAS as first-line treatment and half were given NIL as first-line treatment. Third, for the base case comparison of secondgeneration tyrosine kinase inhibitors to IM TDM, patients in the second-generation tyrosine kinase inhibitor arm had a $50 \%$ chance of receiving either NIL or DAS (per published MarketScan utilization data). ${ }^{7}$ This utilization rate was varied up to $100 \%$ for either NIL or DAS in sensitivity analysis.

Fourth, comparator drug costs were updated to 2016 using three different price bases: (1) Federal Supply Schedule (FSS), ${ }^{10}$ (2) average wholesale acquisition cost (WAC), and (3) lowest WAC. ${ }^{11}$ The price bases were chosen to reflect varying prices among different types of payers, with FSS representing the lowest price schedule and WAC representing a higher price schedule. The WAC pricing is sourced from the US Centers for Medicare and Medicaid Services (CMS)..1 The rate of drug adherence was applied to drug utilization. ${ }^{12}$ Other non-comparator drug prices were included as part of the aggregated inpatient and outpatient costs (per Padula) and updated to 2016 US dollars using the medical service component of the Consumer Price Index. ${ }^{7,13}$

As with the Padula model, a Markov structure was utilized with a 12-month cycle length and a 5-year timeframe (Figure 1) using TreeAge Pro Suite (TreeAge Software Inc, Williamstown, MA). Newly diagnosed CML-chronic phase patient received first-line therapy for one year. Responding patients remained on the first-line therapy for 4 more years, and non-responding patients switched to another therapy. 


\section{TABLE 1 Clinical and Utility Inputs}

\begin{tabular}{|c|c|}
\hline \multicolumn{2}{|c|}{ Major molecular response (12-month) } \\
\hline Imatinib alone $e^{a}$ & 0.37 \\
\hline Imatinib TDM ${ }^{\mathrm{b}}$ & 0.65 \\
\hline Dasatinibc & 0.52 \\
\hline Nilotiinib $^{d}$ & 0.53 \\
\hline \multicolumn{2}{|l|}{ Switch rates ${ }^{e}$} \\
\hline Imatinib to dasatinib & 0.50 \\
\hline Imatinib to nilotinib & 0.50 \\
\hline Nilotinib to dasatinib & 0.95 \\
\hline Nilotinib to imatinib & 0.15 \\
\hline Dasatinib to nilontib & 0.85 \\
\hline Dasatinib to imatinib & 0.15 \\
\hline \multicolumn{2}{|l|}{$\mathrm{AP} / \mathrm{BC}^{\mathrm{e}}$} \\
\hline Imatinib & 0.07 \\
\hline Dasatinib & 0.05 \\
\hline Nilotinib & 0.04 \\
\hline \multicolumn{2}{|l|}{ Overall survivale } \\
\hline Imatinib & 0.74 \\
\hline Dasatinib & 0.75 \\
\hline Nilotinib & 0.30 \\
\hline $\mathrm{AP} / \mathrm{BC}$ & 0.50 \\
\hline \multicolumn{2}{|l|}{ Utility } \\
\hline Baseline utility & 0.75 \\
\hline Treatment response & 0.90 \\
\hline AP/BC (first year) & 0.60 \\
\hline AP/BC (after first year) & 0.85 \\
\hline Treatment switch & 0.58 \\
\hline
\end{tabular}

${ }^{a}$ Rousselot $P$, Johnson-Ansah $H$, Huguet $F$, et al. Personalized daily doses of imatinib by therapeutic drug monitoring increase the rates of molecular responses in patients with chronic myeloid leukemia. Final results of the OPTIM TDM imatinib study in patients with CML. Blood. 2015;126(23):133. ${ }^{b}$ Kantarjian $H$, Shah NP, Hochhaus A, et al. Dasatinib versus imatinib in newly diagnosed chronic-phase chronic myeloid leukemia. N Engl J Med. 2010;362(24):2260-70.

'Kantarjian HM, Shah NP, Cortes JE, et al. Dasatinib or imatinib in newly diagnosed chronic-phase chronic myeloid leukemia: 2-year follow-up from a randomized phase 3 trial (DASISION). Blood. 2012;119(5):1123-9.

'Larson RA, Hochhaus A, Hughes TP, et al. Nilotinib vs imatinib in patients with newly diagnosed Philadelphia chromosome-positive chronic myeloid leukemia in chronic phase: ENESTnd 3-year follow-up. Leukemia. 2012;26(10):2197-203. ePadula WV, Larson RA, Dusetzina SB, et al. Cost-effectiveness of tyrosine kinase inhibitor treatment strategies for chronic myeloid leukemia in chronic phase after generic entry of imatinib in the United States. J Natl Cancer Inst. 2016;108(7):djw003.

$\mathrm{AP} / \mathrm{BC}=$ accelerated phase $/$ blast crisis
Half of non-responding patients receiving IM or IM TDM as first-line therapy were switched to NIL and the other half were switched to DAS. Non-responding patients receiving NIL or DAS as first-line therapy were switched to the other second-generation tyrosine kinase inhibitor or IM in a proportion of $85 \%$ and $15 \%$ respectively, which is based on MarketScan data and expert opinion from the Padula model. Following the switch, the model evaluated patients for 4 more years based on a systemic review of 5-year survival in the Padula model. ${ }^{7}$

As with the original Padula model, if patients progress to accelerated phase or blast crisis $(\mathrm{AP} / \mathrm{BC})$ they were assumed to discontinue tyrosine kinase inhibitor-based therapy and undergo allogeneic transplantation.

Efficacy in the model and rates for therapy switches are based on 12-month major molecular response (MMR) and were taken from published clinical Phase 3 studies for newly diagnosed CML patients comparing IM TDM to IM alone and comparing IM alone to DAS and NIL: IM alone 37\%, IM TDM 65\%, DAS 52\%, NIL 53\% (Table 1). . $^{54-16}$

All other clinical inputs values including survival and death rates are taken directly from the Padula model. IM TDM clinical inputs other than MMR are assumed to be equal to those for IM alone in the Padula model.

Health utilities for each health state were extracted from a published community-rated CML health-state study (Table 1). ${ }^{17}$ Baseline health utility was assumed to remain unchanged at 0.75 QALYs for newly diagnosed CML patients until the 12-month time point. Responding patients resulted in a higher health utility state (0.90 QALYs). As in the Padula model, the rate of $\mathrm{AP} / \mathrm{BC}$ was low (7\% for IM, $5 \%$ for DAS, and $4 \%$ for NIL) with a high one-year mortality of $50 \%$. Utility for these patients was low in first year (0.60 QALYs) but improved as patients were followed for the remaining duration of the study with improving utility (0.85 QALYs). Utility inputs were used to calculate quality-adjusted life-year (QALY) endpoints, which were discounted at 3\% annually.

Tyrosine kinase inhibitor drug costs used three different price bases: (1) FSS, ${ }^{10}$ (2) average WAC, ${ }^{11}$ and (3) lowest WAC ${ }^{11}$ (Table 2). These price bases represent ranges of cost/mg drug pricing for each of the three comparators across the price bases, where from the lowest basis FSS to the highest basis average WAC, IM prices ranges $\$ 0.12 / \mathrm{mg}$ to $\$ 0.59 / \mathrm{mg}$ (391\%), DAS ranges $\$ 2.47 / \mathrm{mg}$ to $\$ 4.20 / \mathrm{mg}(70 \%)$, and NIL ranges $\$ 0.40$ to $\$ 0.59$ (45\%).

A drug adherence rate of $76.3 \%$ was applied to the approved labelled drug utilization rates for all CML drugs based on a published study in CML drug adherence. ${ }^{12}$ Adherence rates were not applied to efficacy parameters as the impact of real-world adherence could not be reliably 


\section{TABLE 2 Cost Inputs}

\begin{tabular}{|c|c|c|c|c|c|c|}
\hline \multirow[b]{3}{*}{ Comparator drug costs ${ }^{a}$} & \multicolumn{2}{|c|}{ FSS Average } & \multicolumn{4}{|c|}{ WAC } \\
\hline & \multirow[b]{2}{*}{$\$ / m g$} & \multirow[b]{2}{*}{ Annual, \$ } & \multicolumn{2}{|c|}{ Low } & \multicolumn{2}{|c|}{ Average } \\
\hline & & & $\$ / m g$ & Annual, \$ & $\$ / m g$ & Annual, \$ \\
\hline \multicolumn{7}{|l|}{ Imatinib } \\
\hline Generic & 0.12 & 13,406 & 0.39 & 43,963 & 0.59 & 65,848 \\
\hline Brand & 0.69 & 76,826 & 0.84 & 93,967 & 0.87 & 97,416 \\
\hline Dasatinib (Brand) & 2.47 & 68,721 & 2.88 & 80,091 & 4.20 & 116,868 \\
\hline \multirow[t]{2}{*}{ Nilotinib (Brand) } & 0.40 & 67,532 & 0.50 & 84,083 & 0.59 & 98,097 \\
\hline & \multicolumn{2}{|c|}{ FSS } & \multicolumn{2}{|c|}{ WAC } & \multicolumn{2}{|c|}{ WAC } \\
\hline Other annual costs & \multicolumn{2}{|c|}{ Average, \$ } & \multicolumn{2}{|c|}{ Low, $\$$} & \multicolumn{2}{|c|}{ Average, \$ } \\
\hline \multicolumn{7}{|l|}{ Imatinib } \\
\hline Direct outpatient costs ${ }^{\mathrm{b}, \mathrm{c}}$ & \multicolumn{2}{|c|}{9,098} & \multicolumn{2}{|c|}{9,098} & \multicolumn{2}{|c|}{9,098} \\
\hline Direct inpatient costs $s^{b, c}$ & \multicolumn{2}{|c|}{17,259} & \multicolumn{2}{|c|}{17,259} & \multicolumn{2}{|c|}{17,259} \\
\hline Direct drug payments ${ }^{a}$ & \multicolumn{2}{|c|}{13,406} & \multicolumn{2}{|c|}{43,963} & \multicolumn{2}{|c|}{65,848} \\
\hline Total annual costs & \multicolumn{2}{|c|}{39,764} & \multicolumn{2}{|c|}{70,321} & \multicolumn{2}{|c|}{92,206} \\
\hline \multicolumn{7}{|l|}{ Dasatinib } \\
\hline Direct outpatient costs ${ }^{b, c}$ & \multicolumn{2}{|c|}{9,173} & \multicolumn{2}{|c|}{9,173} & \multicolumn{2}{|c|}{9,173} \\
\hline Direct inpatient costs $s^{b, c}$ & & & & & & \\
\hline Direct drug payments $^{a}$ & & & & & & \\
\hline Total annual costs & & & & & & \\
\hline Nilotinib & & & & & & \\
\hline Direct outpatient costs ${ }^{b, c}$ & & & & & & \\
\hline Direct inpatient costs $s^{b, c}$ & & & & & & \\
\hline Direct drug payments ${ }^{a}$ & & & & & & \\
\hline Total annual costs & & & & & & \\
\hline Allogenic transplant $\mathrm{t}^{\mathrm{b}, \mathrm{c}}$ & & & & & & \\
\hline
\end{tabular}

${ }^{a}$ www.buyandbill.com

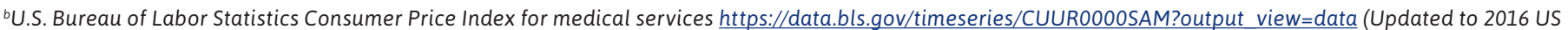
dollars).

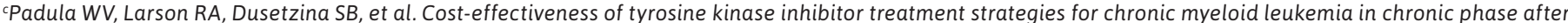
generic entry of imatinib in the United States. J Natl Cancer Inst. 2016;108(7):djw003.

FSS = Federal Supply Schedule; $W A C=$ wholesale acquisition costs.

applied to the clinical trial based efficacy inputs. The cost of TDM for IM was added to the IM TDM comparator arm at \$228 annually. Other costs in the model including annual direct inpatient and outpatient costs and the cost of allogenic transplant were taken from the Padula model based on an aggregated MarketScan analysis ${ }^{7}$ and updated to 2016 US dollars using the medical services component of the Consumer Price Index ${ }^{13}$ and discounted at $3 \%$ annually (Table 2).

The model calculated the following outcomes over 5 years: total costs, QALYs, and cost-effectiveness (cost per
QALY). Subanalysis was also conducted on savings per IM TDM 5-yr responder which describes the cost to payers of getting a first-line therapeutic response. Comparisons were made between IM TDM versus IM alone and between IM TDM versus DAS/NIL. Deterministic and probabilistic sensitivity analyses were conducted for all variables. Clinical and utility ranges published in the Padula model were utilized, and for comparator drug costs, FSS was used as the low range and WAC average as the high range. Also, the rate of patients entering into the second-generation tyrosine kinase inhibitor arm was varied from the 50\%/50\% 


\section{TABLE 3 Base Case Results (over 5 years)}

\begin{tabular}{|c|c|c|c|c|c|c|c|}
\hline \multirow[b]{3}{*}{ Treatment } & \multicolumn{3}{|c|}{ Total cost } & \multirow[b]{3}{*}{ QALY } & \multicolumn{3}{|c|}{ Total cost per first-line responder } \\
\hline & \multirow[b]{2}{*}{ FSS average } & \multicolumn{2}{|c|}{ WAC } & & \multirow[b]{2}{*}{ FSS average } & \multicolumn{2}{|c|}{ WAC } \\
\hline & & Low & Average & & & Low & Average \\
\hline IM TDM & 233,965 & 350,090 & 446,205 & 3.82 & 448,200 & 670,670 & 854,799 \\
\hline IM alone & 270,905 & 366,966 & 461,657 & 3.57 & 732,176 & 991,800 & $1,247,722$ \\
\hline Difference (IM alone minus IM TDM) & 36,940 & 16,876 & 15,452 & -0.25 & 283,967 & 321,130 & 392,923 \\
\hline DAS/NIL & 406,385 & 467,106 & 575,606 & 3.74 & 778,515 & 894,839 & $1,102,693$ \\
\hline Difference (DAS/NIL minus IM TDM) & 172,420 & 117,016 & 129,401 & -0.08 & 330,307 & 224,169 & 247,895 \\
\hline
\end{tabular}

DAS = dasatinib; FSS =Federal Supply Schedule; $N I L=$ nilotinib; $W A C=$ wholesale acquisition costs.

assumption for DAS/NIL up to $100 \%$ for each drug. Other costs were varied by a standard $20 \%$ above and below the base case. Multivariable probabilistic sensitivity analysis was performed using 10,000 Monte Carlo simulations; gamma distributions were applied to costs, and beta distributions for probabilities and utilities. Randomly selected parameter values from their assumed distributions were used in order to provide more realistic CML population results.

\section{Results}

The base case results (Table 3) for first-line treatment of CML over 5 years show QALY gains with IM TDM were 0.25 greater than with IM alone, or a 7.0\% increase from 3.57 to 3.82 QALYs. Total cost savings with IM TDM over IM alone ranged from $\$ 15,452$ (3.3\%) with average WAC pricing to $\$ 36,940$ (13.6\%) with FSS pricing. Thus, IM TDM is more cost effective than IM alone, and since IM TDM has greater effectiveness and lower costs versus IM alone, it is a dominant treatment strategy in this comparison.

QALYs gained were 0.08 greater with IM TDM over 5 years versus NIL/DAS, or a $2.1 \%$ increase. Total cost savings with IM TDM ranged from \$117,006 (25.1\%) with Low WAC pricing to $\$ 172,420$ (42.4\%) with FSS pricing. Thus, IM TDM is a dominant treatment strategy since IM TDM has greater effectiveness and lower costs than NIL/DAS.

In terms of the cost per first-line responder over 5 years, IM TDM costs ranged from $\$ 283,967$ lower than IM alone using FSS average pricing to $\$ 561,252$ lower than IM alone using WAC average pricing. Versus NIL/DAS, IM TDM costs ranged from $\$ 330,307$ lower FSS average pricing to $\$ 416,224$ lower using WAC average pricing.
All results were confirmed as robust by deterministic sensitivity analyses, where no univariate changes inputs caused IM TDM no lose dominance over IM alone or DAS/ NIL. Key results included that the price of generic imatinib can vary well beyond the three different price bases used in the base case without changing the result showing that IM TDM has lower total costs than the comparators. In fact, the price of generic imatinib could increase significantly from the base case value before IM TDM total costs are greater than DAS/NIL total costs: 77\% increase in WAC low price, $58 \%$ increase in WAC average price and $477 \%$ increase in FSS price. Also, the MMR for IM TDM could decrease from a base case of $65 \%$ to $55 \%$ before IM TDM is no longer dominant over DAS/NIL, and it could drop to $58 \%$ before IM TDM is no longer dominant over IM alone. Other clinical inputs such as survival rates and utilities did not demonstrate large impact on the results in the sensitivity analysis.

Further, changing drug utilization rates, including varying the adherence rates, in the DAS/NIL arm did not significantly impact the results. Using either only NIL or only DAS in the DAS/NIL arm (in contrast to the 50\%/50\% utilization in the base case) did not change the dominance of IM TDM over DAS/NIL. Lastly, wide variations in the cost of TDM and changes in the base case $3.0 \%$ discount rate did not change the dominance of IM TDM over IM alone and DAS/NIL.

The probabilistic sensitivity analysis (Figure 2) suggested that IM TDM was overwhelmingly cost-effective compared with DAS/NIL. IM TDM dominated DAS/NIL with greater utility and lower costs in $88.1 \%$ of simulations. An additional $10.9 \%$ of simulations identified IM TDM as cost-effective, that is, below a willingness-to-pay threshold of $\$ 100,000$ per QALY. Thus, only $1.0 \%$ of simulations rose above the threshold line in Figure 2. Further, over 99.9\% of simulations determined that IM TDM had improved net monetary 


\section{FIGURE 2 Willingness to Pay (at \$100,000/QALY threshold) IM TDM vs DAS/NIL}

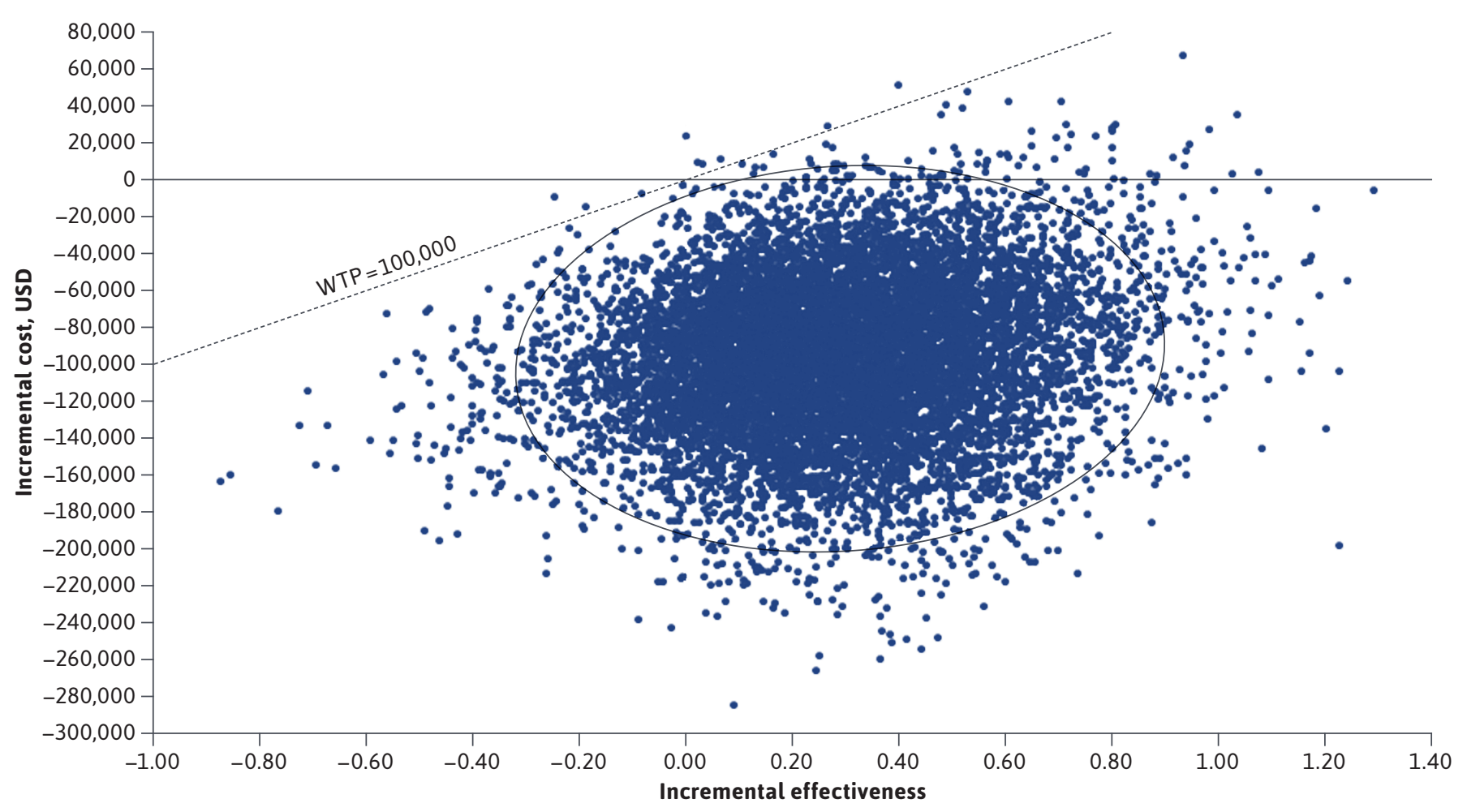

DAS= dasatinib; $N I L=$ nilotinib; $I M T D M=$ imatinib with therapeutic drug monitoring; $W T P=$ willingness to pay.

benefit. Probabilistic sensitivity analysis using other price bases yield similar results.

\section{Discussion}

While second-generation tyrosine kinase inhibitor in CML have been used as first-line therapy in CML due to superior effectiveness compared to first-generation tyrosine kinase inhibitor IM, recent findings suggest that IM TDM has greater efficacy than second-generation tyrosine kinase inhibitor. ${ }^{5}$ The potential economic benefit of this greater efficacy with IM TDM has not been studied previously. Thus, this study is the first cost-effectiveness analysis of generic IM TDM in CML.

The study base case results showing IM TDM to be dominant over both IM alone and DAS/NIL suggest that IM TDM is both a clinically and economically viable first-line treatment option for patients with CML. The greater QALYs are driven primarily by IM TDM's higher efficacy putting patients into health states with greater quality of life. The lower costs for IM TDM over IM alone are, again, primarily the result of IM TDM improved efficacy, where IM TDM patients are in health states with lower costs. Also, the low cost of TDM itself relative to the total costs has little impact on the cost comparison. In the IM TDM versus DAS/ NIL comparison, the lower price of generic imatinib is the primary driver of the cost difference.

The base case does cover a wide range of drug price scenarios that reflects various settings in the US market. The results with IM TDM being dominant are consistent across all of these scenarios, with the FSS price basis showing the greatest cost disparity among the comparators.

While the three drug pricing bases used in the model base case do have a large range of prices, they do not include all of the possible drug pricing scenarios in the market. Various drug pricing contracts relevant to payers and providers are not explicitly included in the model as they are typically contracted confidentially and are not publicly available. However, the various pricing scenarios using different price bases with varying relative price 
differences among comparators and sensitivity analyses, such as used in this model, do show that the results are robust over a large range of price scenarios. Lastly, a comparison of the model's price bases with a recently published study of generic imatinib list prices using MarketScan Commercial Research Database found the pricing results in that study to be similar to high range WAC pricing inputs used in this model, and thus, validated the imatinib pricing inputs used. ${ }^{9}$

This study can be seen as a followup and extension of the previously published Padula model which showed IM alone to be a cost-effective firstline treatment versus DAS/NIL. However, this study's results differ from the Padula model in two major ways. First, while the Padula model was conducted at a time when the CML market had yet to include realworld, post-loss of exclusivity generic imatinib pricing, our model results do reflect post-loss of exclusivity generic imatinib pricing. And second, while the Padula model demonstrated cost-effectiveness for IM alone versus both DAS and NIL, our model showed dominance of IM TDM versus IM alone and DAS/NIL.

Another US cost-effectiveness model of 16 tyrosine kinase inhibitor treatment combinations in CML found one combination using first-line imatinib and one combination using a first-line second-generation tyrosine kinase inhibitor to be cost-effective using a lifetime timeframe. ${ }^{9}$ However, neither of these first-line treatments nor any of the other 16 CML treatment combinations studies were found to be dominant to the other treatments, and the study used a much higher threshold to define cost-effectiveness than used in this study. Using the $\$ 100,000$ cost/QALY threshold of this study, none of the 16 treatment combinations from this earlier study would be considered cost-effective.
Thus, our study clearly demonstrated the IM TDM is a dominant CML treatment at $\$ 50,000 / \mathrm{QALY}$ threshold over 5 years while other studies have used longer time frames, higher thresholds and only shown cost-effectiveness.

\section{LIMITATIONS}

This study is limited to a 5-year time horizon. Longer time horizons would involve the use of various clinical and economic parameters whose values are unknown at this time and would be difficult to estimate with a reasonable level of uncertainty.

As with the original Padula model, this study is limited by the lack of data regarding additional switches for tyrosine kinase inhibitors beyond three- or 12-month efficacy/tolerability endpoints as data is limited to available published clinical trials. However, this limitation does not likely significantly bias the model results in favor of or against any of the comparators.

\section{Conclusions}

The analysis suggests that IM TDM is both a clinically and economically viable first-line treatment option for CML. Not only can IM TDM potentially improve clinical outcomes, it can also lower costs from the existing standard of care.

\section{DISCLOSURES}

This study was funded by Saladax Biomedical. Salamone is an employee of Saladax Biomedical. This study was presented at the IATDMCT Congress, September 2018, Brisbane, Australia.

\section{REFERENCES}

1. Chopade P, Akard LP. Improving outcomes in chronic myeloid leukemia over time in the era of tyrosine kinase inhibitors. Clin Lymphoma Myeloma Leuk. 2018;18(11):710-12.
2. Saikia T. The cure of chronic myeloid leukemia: Are we there yet? Curr Oncol Rep. 2018;20(2):12.

3. Yu H, Steeghs N, Nijenhuis CM, Schellens JHM, Beijnen JH, Huitema ADR. Practical guidelines for therapeutic drug monitoring of anticancer tyrosine kinase inhibitors: focus on the pharmacokinetic targets. Clin Pharmacokinet. 2014;53(4):305-25.

4. Verheijen RB, Yu H, Schellens JHM, Beijnen, JH, Steeghs, N, Huitema, ADR. Practical recommendations for therapeutic drug monitoring of kinase inhibitors in oncology. Clin Pharmacol Ther. 2017;102(5):765-76.

5. Rousselot $\mathrm{P}$, Johnson-Ansah $\mathrm{H}$, Huguet $F$, et al. Personalized daily doses of imatinib by therapeutic drug monitoring increase the rates of molecular responses in patients with chronic myeloid leukemia. Final results of the OPTIM TDM imatinib study in patients with CML. Blood. 2015;126(23):133.

6. Conti RM, Berndt ER. Specialty drug prices and utilization after loss of U.S. patent exclusivity, 2001-2007. NBER Working Paper No. w20016. 2014

7. Padula WV, Larson RA, Dusetzina SB, et al. Cost-effectiveness of tyrosine kinase inhibitor treatment strategies for chronic myeloid leukemia in chronic phase after generic entry of imatinib in the United States. J Natl Cancer Inst. 2016;108(7):djw003.

8. Dusetzina SB, Winn AN, Abel GA, Huskamp HA, Keating NL. Cost sharing and adherence to tyrosine kinase inhibitors for patients with chronic myeloid leukemia. J Clin Oncol. 2014;32(4):306-11.

9. Rochau U, Kluibenschaedl M, Stenehjem D, et al. Effectiveness and cost-effectiveness of sequential treatment of patients with chronic myeloid leukemia in the United States: A decision analysis. Leuk Res Treatment. 2015;2015:982395.

10. U.S. Department of Veterans Affairs. Pharmaceutical prices. Accessed May 15, 2017. https://www.va.gov/opal/nac/fss/ pharmPrices.asp 
11. BuyandBill.com HCPCS Unit-Level Drug Pricing. Accessed May 15, 2017. www.buyandbill.com

12. Tsang J, Rudychev I, Pescatore S. Prescription compliance and persistency in chronic myeloid leukemia (CML) and gastrointestinal stromal tumor (GIST) patients (pts) on imatinib (IM). J Clin Oncol. 2006;24(S18):6119.

13. U.S. Bureau of Labor Statistics. Medical Consumer Price Index. https://data.bls.gov/timeseries/ CUUR0000SAM?output_view=data
14. Kantarjian H, Shah NP, Hochhaus A, et al. Dasatinib versus imatinib in newly diagnosed chronic-phase chronic myeloid leukemia. N Engl J Med. 2010;362(24):2260-70.

15. Kantarjian HM, Shah NP, Cortes JE, et al. Dasatinib or imatinib in newly diagnosed chronic-phase chronic myeloid leukemia: 2-year follow-up from a randomized phase 3 trial (DASISION). Blood. 2012;119(5):1123-29.

16. Larson RA, Hochhaus A, Hughes TP, et al. Nilotinib vs imatinib in patients with newly diagnosed Philadelphia chromosome-positive chronic myeloid leukemia in chronic phase: ENESTnd 3-year followup. Leukemia. 2012;26(10):2197-203.
17. Szabo SM, Levy AR, Davis C, Holyoake TL, Cortes J. A multinational study of health state preference values associated with chronic myelogenous leukemia. Value Health. 2010;13(1):103-11.

18. U.S. Department of Veterans Affairs. Pharmaceutical prices. Accessed on May 15, 2017. https://www.va.gov/opal/nac/ fss/pharmPrices.asp 